\title{
Incremental Enhanced Artificial Bee Colony Algorithm with Local Search
}

\author{
Neha Pathak \\ Research Scholar, Computer \\ Science Dept. \\ ITM UNIVERSE \\ Gwalior, India
}

\author{
Manuj Mishra \\ Asst. Professor, Computer \\ Science Dept. \\ ITM UNIVERSE \\ Gwalior, India
}

\author{
Shiv Pratap Singh Kushwah \\ Asst. Professor, Computer \\ Science Dept \\ ITM UNIVERSE \\ Gwalior,India
}

\begin{abstract}
Swarm intelligence is the collective problem solving behavior of groups of artificial agents. These agents local interaction with each other can be negative, positive or neutral. Here positive interaction helps agents to solve a problem while negative interaction block the agents for solving problem and neutral interaction does not affect the swarm's performance. In this work, incremental enhanced $\mathrm{ABC}$ algorithm with local search is used for reducing negative interaction without complexifying the agent's behavior. Here in the enhanced artificial bee colony algorithm, one additional phase in the form of mutation operator is used. With the help of mutation operator, algorithm may not be trapped into local optima due to the chance of changing local best position. The experimental results show that the performance of proposed algorithm and the proposed algorithm is compared with standard $\mathrm{ABC}$ algorithm, Artificial Bee Colony algorithm with mutation algorithm.
\end{abstract}

\section{Keywords}

Artificial Bee Colony, ABC, Genetic Algorithm, Mutation, incremental social learning, Swarm Intelligence.

\section{INTRODUCTION}

The problem of optimization is one of the most crucial problems now a day and lots of work have been done previously to solve these problems. There are many types of optimization algorithm like GA, ABC, PSO, ACO[4], etc. and lots of works in these algorithms are available in the literature. In paper [1], Dervis Karaboga defined an algorithm, called Artificial Bee Colony (ABC) for solving numerical optimization problem. This algorithm is based on the foraging behavior of honey bees. Different versions of $\mathrm{ABC}$ algorithm have been proposed like $\mathrm{ABC}$ with mutation, $\mathrm{ABC}$ with crossover, $\mathrm{ABC}$ with SPV , $\mathrm{ABC}$ with crossover and mutation and many different versions have been proposed. In paper [2], dynamically division of bees into subgroup and the searching process is performed on these generated subgroups. In paper [3], Karaboga defines the modified algorithm for solving real parameter optimization problems and comparative study on different evolutionary algorithms like particle swarm optimization (PSO), ABC algorithm, Differential evolution (DE), Ant colony algorithm (ACO) etc and performance derived on different benchmark functions, are discussed on paper [5,7]. For improving the exploitation, karaboga proposed a modified $\mathrm{ABC}$ algorithm based on global best solution in paper [6]. D. Haijun and F. Qingxian proposed a modified artificial bee colony algorithm for function optimization in paper [8].

In paper [10], travelling salesman problem using bee colony with SPV rule is used. In this paper, incremental approach is used in enhanced $\mathrm{ABC}$ algorithm for improving local search.
The overall paper is organized as follows. Section 2 describes the artificial bee colony algorithm with mutation. In section 3 , incremental Artificial Bee Colony algorithm with local search is explained. Travelling salesman problem is discussed in section 4. Section 5 describes experimental results and parameter setup for result comparison. Finally, section 6 concludes the paper.

\section{ARTIFICIAL BEE COLONY ALGORITHM WITH MUTATION}

In paper [9], one more phase in the form of mutation operator is added to original Artificial Bee Colony algorithm. In standard $\mathrm{ABC}$ algorithm, there are only 4 phases that described the overall working of this algorithm, but here one additional phase after the scout bee phase of $\mathrm{ABC}$ algorithm is added in the form of Mutation operator. Now modified artificial bee colony algorithm has five phases: first initialization phase then employed bee phase, onlooker bee phase, scout bee phase and finally mutation phase. For the local search, employed bee phase is used and the mutation phase is used to find out the new search area of the solution space. With the help of mutation operator, there may be a possibility to change the local best position and the algorithm may not be trapped into local optima. In this work, the mutation phase is implemented on the probabilistic way in each iteration for searching food source during the life process of $\mathrm{ABC}$ optimization technique. Food sources are selected randomly from the food size to perform mutation operation. In mutation phase, if generated offspring's fitness is greater than the older one then replaces the older offspring's from the new one. Uniform mutation is used in this work.

The overall algorithm is described in following steps:

- Initialization phase.

- REPEAT

(a) In the Memory, Employed bees are placed on the food sources;

(b) Generate new offspring from older offspring after Applying mutation operator.

(c) In the memory, onlooker bees are placed on the food sources;

(d) For finding new food sources, Send the scout bee to the search area

- UNTIL (requirements not met).

\section{INCREMENTAL ABC ALGORITHM WITH LOCAL SEARCH}

In the original artificial bee colony algorithm [11], food source position represents a solution and its nectar amount represents fitness value. The employed and onlooker bee 
phase finds better food position and scout bee phase is used to generate new food sources if all the food sources exhausts.

In the incremental artificial bee colony algorithm, Food sources are increased with a control parameter ' $\mathrm{g}$ ', that means population size is increased in incremental $\mathrm{ABC}$ algorithm. In this, for every ' $\mathrm{g}$ ' iterations, a new food source is increased until maximum number of food sources is reached. IABC algorithm begins with some food sources and placed new food sources towards best-so-far solution. The above concept is implemented as -

$\mathrm{X}_{\text {new }, \mathrm{i}}^{\prime}=\mathrm{X}_{\text {new }, \mathrm{i}}+\operatorname{rand}[0,1]\left(\mathrm{X}_{\text {gbest }, \mathrm{i}}-\mathrm{X}_{\text {new }, \mathrm{i}}\right)$

Where $X_{\text {new }, \mathrm{i}}=$ new food source locations generated randomly

$X_{\text {new }, \mathrm{i}}^{\prime}=$ updated new food source location

$\mathrm{X}_{\text {gbest, } \mathrm{I}}=$ best-so-far locations of food sources

Similarly in scout bee phase. Replacement policies are used like above by using a control parameter ' $R_{\text {factor'. This }}$ parameter controls the closeness of new food source with best-so-far food source. The above concept is implemented as-

$X_{\text {new }, \mathrm{i}}^{\prime}=X_{\text {gbest }, \mathrm{i}}+\mathrm{R}_{\text {factor }}\left(X_{\text {gbest }, \mathrm{i}}-\mathrm{X}_{\text {new }, \mathrm{i}}\right)$

The above modifications improve the exploit behavior and help to converge towards optimal solution.

Basic steps of incremental $\mathrm{ABC}$ algorithm are given below-

1. Initialize number of food sources

2. REPEAT

If Failure_attempts $=$ Failure_Max then

Perform local search on the food sources selected randomly.

Else

Perform local search on the best food source

3. Employed bee phase

4. Scout bee phase

If new food source addition criteria met then

Add new food source

5. UNTIL ( termination criteria not met)

\section{TRAVELLING SALESMAN PROBLEM}

The travelling salesman (TSP) problem is described as follows: Given a list of cities and a travelling cost between each pair of cities. Here the TSP problem is that to find the shortest route with minimum cost between all the cities and returning to starting position.

In figure 1, there are 7 cities which are connected and $\mathrm{A}$ is the source node or starting position. Here from the starting node, visit all the cities with minimum cost. Cost between two cities is not mentioned here. It may vary from problem to problem.

To calculate the minimum cost between all the cities from initial node, following formula is used.

$$
\text { Cost }=\sum_{1}^{n} C_{i j}
$$

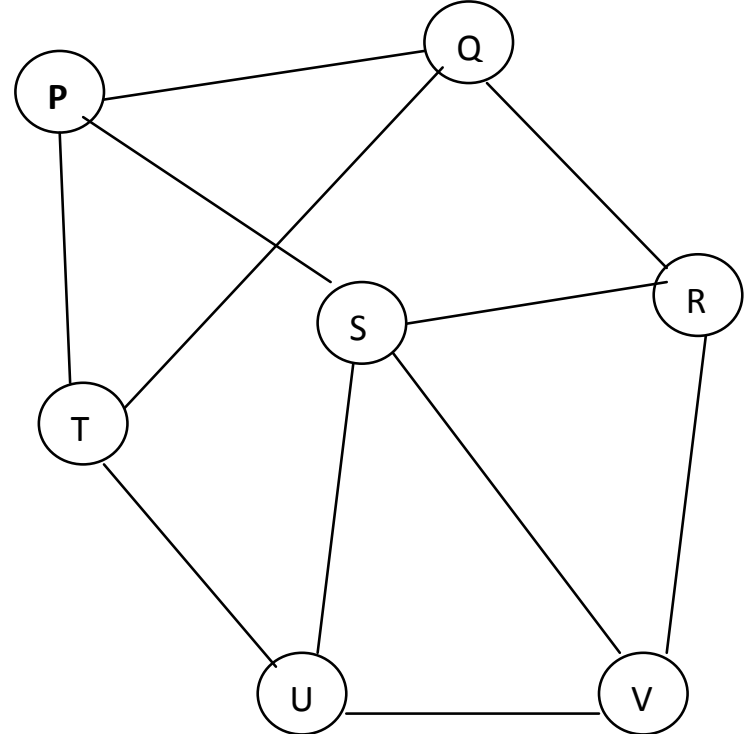

Source node

Figure 1: Graph representation of TSP Problem

\section{PROPOSED WORK}

In this proposed work, incremental modified artificial bee colony algorithm with local search is used. In the enhanced artificial bee colony algorithm, one additional phase in the form of mutation operator is used. Here mutation operator is used after the employed bee phase of original $\mathrm{ABC}$ algorithm. Now modified ABC algorithm has five phases: Initialization phase, employed bee phase, mutation phase, onlooker bee phase and scout bee phase. When performing mutation, select randomly one of the food sources and replace its one of the dimension value by random number generated in between lower and upper bound value of the food source. The incremental approach that is used in this work for improving local search is explained in section 3 . The proposed algorithm is described below-

1. Initialize number of food sources

2. REPEAT

If Failure_attempts $=$ Failure_Max then

Perform local search on the food sources selected randomly.

Else

Perform local search on the best food source

3. Employed bee phase

4. Mutation phase

5. Scout bee phase

If new food source addition criteria met then

Add new food source

a. UNTIL ( termination criteria not met)

In the above algorithm, mutation phase is explained below -

\section{MUTATION PHASE}

If mutation criteria is met then

(a) From current population, select random particle for mutation operator. 
(b) After applying mutation operation, new individuals generated.

(c) New feasible solution generated as a result of mutation operation.

(d) Calculate the cost for that individual.

(e) Calculate updated individual fitness.

\section{EXPERIMENTAL RESULTS AND PARAMETER SETUP}

For interpreting Travelling salesman problem by using incremental enhanced $\mathrm{ABC}$ algorithm with local search, the following control parameters are used -

Maximum cycle number, i.e. $\mathrm{MCN}=500,1000,1500$ and 2000

Colony size $=20$.

Each individual Dimension $=10,20$ and 30

Number of Iteration $=30$.

Experimental results are shown in fig 1, fig 2 and fig 3 for the dimensions 10, 20 and 30 respectively.

\section{CONCLUSION}

Artificial bee colony algorithm is one of the optimization algorithms for interpreting different optimization problems which are used to find out the global optima. In this work, enhanced algorithm is used which uses one additional step in the form of mutation operator is used. With this mutation operator, Algorithm may not be trapped into local optima. Finally enhanced $\mathrm{ABC}$ algorithm with local search using incremental approach is used and the proposed work is compared with previously defined algorithms and implemented on travelling salesman problem. Future work is to used the proposed work on different types of optimization problem and compare them with older one.

\section{REFERENCES}

[1] 'D. Karaboga," An idea based on honey bee swarm for numerical optimization" Techn. Rep. TR06,Erciyes Univ. Press, Erciyes, 2005.

[2] Shraddha Saxena, Kavita Sharma, Savita Shiwani and Harish Sharma, "Lbest Artificial Bee Colony using Structured Swarm", Advance Computing Conference (IACC), 2014 IEEE, pp-1354-1360.
[3] B. Akay and D. Karaboga," A modified artificial bee colony algorithm for real-parameter optimization" Information Sciences, doi:10.1016/j.ins.2010.07.015, 2010.

[4] M. Dorigo and G. Di Caro," Ant colony optimization: a new meta-heuristic" In Evolutionary Computation, 1999. CEC 99. Proceedings of the 1999 Congress on, volume 2. IEEE, 1999.

[5] J. Vesterstrom and R. Thomsen," A comparative study of differential evolution, particle swarm optimization, and evolutionary algorithms on numerical benchmark problems" In Evolutionary Computation, 2004. CEC2004. Congress on, volume 2, pages 1980-1987. IEEE, 2004.

[6] G. Zhu and S. Kwong," Gbest-guided artificial bee colony algorithm for numerical function optimization" Applied Mathematics and Computation, 217(7):31663173, 2010.

[7] D. Karaboga and B. Akay," A comparative study of artificial bee colony algorithm" Applied Mathematics and Computation, 214(1):108-132, 2009.

[8] D. Haijun F. Qingxian,"Bee colony algorithm for the function optimization". Science Paper Online,08:448 456, August 2008.

[9] Amit Singh, Neetesh Gupta and Amit Singhal, "Artificial bee colony algorithm with uniform mutation", Proceedings of the International Conference on Soft Computing for Problem Solving (SocProS 2011) December 20-22, 2011, Volume 130, 2012, pp 503-511.

[10] Montes de Oca, M.A., St"utzle, T.: Towards incremental social learning in optimization and multiagent systems. In: Rand, W., et al. (eds.) Workshop on Evolutionary Computation and Multiagent Systems Simulation of the Genetic and Evolutionary Computation Conference (GECCO 2008), pp. 1939-1944. ACM Press, New York (2008).

[11] D. Aydin, T. Liao, Marco A. Montes De Oca and T. Stutzle, "Improving Performance via Population Growth and Local Search: The Case of the Artificial Bee Colony Algorithm" IRIDIA - Technical Report Series TR/IRIDIA/2011-015, ISSN 1781-3794, Aug 2011.

Table1: Comparative Results For Dimension = 10

\begin{tabular}{|c|c|c|c|c|}
\hline Algorithm & MCN = 500 & MCN = 1000 & MCN = 1500 & MCN = 2000 \\
\hline ABC & 88.73 & 83.21 & 81.19 & 79.88 \\
\hline ABC with mutation & 86.90 & 82.65 & 80.09 & 78.15 \\
\hline Proposed Algorithm & 86.12 & 81.57 & 79.24 & 77.92 \\
\hline
\end{tabular}

Table 2: Comparative Results For Dimension $=20$

\begin{tabular}{|c|c|c|c|c|}
\hline Algorithm & MCN = 500 & MCN = 1000 & MCN = 1500 & MCN = 2000 \\
\hline ABC & 175.56 & 172.25 & 169.36 & 167.65 \\
\hline
\end{tabular}


International Journal of Computer Applications (0975 - 8887) Volume 116 - No. 13, April 2015

\begin{tabular}{|c|c|c|c|c|}
\hline ABC with Mutation & 174.25 & 171.11 & 168.68 & 166.21 \\
\hline Proposed Algorithm & 173.58 & 170.65 & 167.49 & 165.39 \\
\hline
\end{tabular}

Table 3: Comparative Results For Dimension $=30$

\begin{tabular}{|c|c|c|c|c|}
\hline Algorithm & MCN = 500 & MCN = 1000 & MCN = 1500 & MCN = 2000 \\
\hline ABC & 268.31 & 265.90 & 263.29 & 261.94 \\
\hline ABC with Mutation & 267.93 & 264.55 & 262.37 & 260.82 \\
\hline Proposed Algorithm & 266.56 & 263.14 & 261.74 & 259.47 \\
\hline
\end{tabular}

